\title{
Environmental Assessment of Fires in Products Using the Fire-LCA Model
}

\author{
MARGARET SIMONSON ${ }^{1}$, PETRA ANDERSSON ${ }^{1}$, PER BLOMQVIST $^{1}$, and \\ HÅKAN STRIPPLE ${ }^{2}$ \\ ${ }^{1}$ Fire Technology \\ SP Swedish National Testing and Research Institute \\ Box 857 \\ SE-501 15 Borås, Sweden \\ 2 IVL-Swedish Environmental Research Institute \\ Box 5302 \\ SE-400 14 Gothenburg, Sweden
}

\begin{abstract}
The environmental impact of products has been increasingly discussed in recent years. In response to questions of the environmental appropriateness of, for example, technologies used to attain a high level of fire safety, a model has been developed to quantify the emissions from fires and include this into a holistic treatment of the environmental impact of a product from the cradle to the grave. This model is based on Life-Cycle Assessment (LCA) methodology but represents a significant improvement over traditional LCA models that cannot include accidents such as fires. The Fire-LCA model has been tested in three case studies: TV, cables, and furniture. This article presents the model in detail, in particular focusing on the difference between the Fire-LCA model and traditional LCA models, using examples from the case studies.
\end{abstract}

KEYWORDS: modeling, statistics, environment, LCA

\section{INTRODUCTION}

Environmental issues are a vital part of our society and the ability to perform accurate estimates and evaluations of environmental parameters are vital tools as we work to improve the environment. Traditionally, environmental studies have focussed mainly on various point emission sources, such as factory chimneys, exhaust gases from vehicles, effluent waters from factories etc. However, in the 1980's it became apparent that a simple measurement of an emission did not provide a full picture of the environmental impact of a specific product or process. The emissions from a chimney, for example, only reflect one of several process steps in the production of a specific product. To fully describe the environmental situation, the entire process chain has to be described including raw material extraction, transport, energy and electric power production, production of the actual product, the waste handling of the product etc. There was, therefore, an obvious need for a new methodology and an analytical tool able to handle this new situation. The tool that was developed during this period (end of 1980's and 1990’s) was: Life-Cycle Assessment (LCA).

However, the Life-Cycle Assessment methodology also needs continuous improvements to handle new aspects of our society and processes. An LCA typically describes a process during normal operation and abnormal conditions such as accidents are left out of the analysis, usually due to lack of a consistent methodology or relevant data. For example, LCA data for power production usually assumes normal running conditions without any 
accidents although one could include provisions for certain accidents in the analysis of the life-cycle provided these could be specified in sufficient detail and occurred with sufficient regularity to make their inclusion relevant.

Assuming that a certain number of fire accidents are unavoidable in any dynamic society we are forced to take fires into consideration when defining any balanced environmental perspective. Up until very recently, no concerted effort has been made to objectively weigh various environmental considerations against each other in a broader perspective rather than merely in terms of emissions upon combustion.

In traditional LCA models a higher fire performance is only included as a change in energy and material consumption and no account is made of the positive effect of higher fire performance in the form of fewer and smaller fires. The emissions from fires contribute to the environmental impact from products and should be included in a more complete evaluation of the environmental impact of a product where the fire performance is an important parameter, i.e., in cases where the fire performance is not a critical product performance characteristic (e.g., underground piping) one should not include this in the product LCA.

An LCA model represents the best modern method to determine the environmental impact of a series of choices concerning the life-cycle of any given product, from exploitation of resources to manufacturing, through use to recycling, re-utilisation or disposal. The development of an LCA model that incorporates fire considerations along with the simultaneous development of testing methods, which give input to this LCA model as a part of their standard procedure, represents a powerful assessment tool.

The Fire-LCA project officially started in September 1998 after completion of a Preparatory Study where the Fire-LCA model was defined [1,2]. After completion of this study a number of case studies were proposed including:

- TV Case Study,

- Cables Case Study, and

- Furniture Case Study.

In the first case a TV with high fire performance enclosure material was compared to that with easily ignitable enclosure material [3-6]. The second application of the Fire-LCA model focussed on a comparison between two types of electrical cables, assumed to have essentially the same fire performance [7,8]. The third, and most recent, application of the Fire-LCA model has focussed on a comparison between two types of furniture, one having the minimal fire performance required on mainland Europe and the other with fire performance akin to that required in the UK $[9,10]$. In all of these applications the model has proven to be robust and to adequately establish the relative environmental impact of important emissions and to what extent fire safety is a benefit or burden on the environment. Details of the results of the three case studies can be found elsewhere and this article will focus on defining the modeling tools used and presenting the most important generic findings of this research, using specific examples from the case studies.

\section{LCA METHODOLOGY}

Life-Cycle Assessment (LCA) is a versatile tool to investigate the environmental aspect of a product, a process or an activity by identifying and quantifying energy and material flows for the system. The use of a product or a process involves much more than just the 
production of the product or use of the process. Every single industrial activity is actually a complex network of activities that involves many different parts of society. Therefore, the need for a system perspective rather than a single object perspective has become vital in environmental research. It is no longer enough to consider just a single step in the production. The entire system must be considered.

The Life Cycle Assessment methodology has been developed in order to handle this system approach. A Life Cycle Assessment covers the entire life cycle from "the cradle to the grave" including crude material extraction, manufacturing, transport and distribution, product use, service and maintenance, recycling and final waste handling such as incineration or landfill. In a life cycle assessment a mathematical model of the system is designed. This model is a representation of the real system with various approximations and assumptions. With LCA methodology it is possible to study complex systems where interactions between different parts of the system exist to provide as complete a picture as possible of the environmental impacts of, for example, a production process.

An LCA usually evaluates the environmental situation based on ecological effects and resource use. In a few cases the work environment has also been included. A traditional LCA does not cover the economic or social effects.

International standards for LCA methodology have been prepared by the International Organisation for Standardisation (ISO). The following standards are available today:

- Principles and framework (ISO 14040) [11],

- Goal and scope definition and inventory analysis (ISO 14041) [12],

- Life cycle impact assessment (ISO 14042) [13], and

- Life cycle impact interpretation (ISO 14043) [14].

Generally the method can be divided into three basic steps with the methodology for the first two steps relatively well established while the third step (Impact assessment) is more difficult and controversial. The first two steps are usually referred to as the life cycle inventory (LCI) and can be applied separately without the following impact assessment. In addition to the different steps in the procedure there can also be an interpretation phase. The three basic steps are shown in Fig. 1.

\section{Goal and Scope}

The goal and scope definition consists of defining the study's purpose, its scope, system boundaries, establishing the functional unit, and establishing a strategy for data collection and quality assurance within the study. Any product or service needs to be represented as a system in the inventory analysis methodology. A system is defined as a collection of materially and energetically connected processes (e.g., fuel extraction processes, manufacturing processes or transport processes), which perform some defined function.

The system is separated from its surroundings by a system boundary. The entire region outside the boundary is known as the system environment.

The functional unit is the measure of performance, which the system delivers. The functional unit describes the main function(s) of the system(s) and is therefore a relevant and well-defined measure of the system. The functional unit has to be clearly defined, measurable, and relevant to input and output data. The functional unit may be either a specific product (such as the TV or sofa used in two of the Fire-LCA case studies) or a 
function (such as the unit surface area covered by paint for a defined period of time). It is important that the functional unit contains measures for the efficiency of the product, durability or lifetime of the product and the quality/performance of the product. In comparative studies, it is essential that the systems are compared on the basis of equivalent functional unit.

Other important aspects to consider in the goal and scope definition include: whether the LCA is complete or if some component is excluded from the study, which type of environmental impact is considered in the study, and a description of other important assumptions.

Life cycle assessment framework

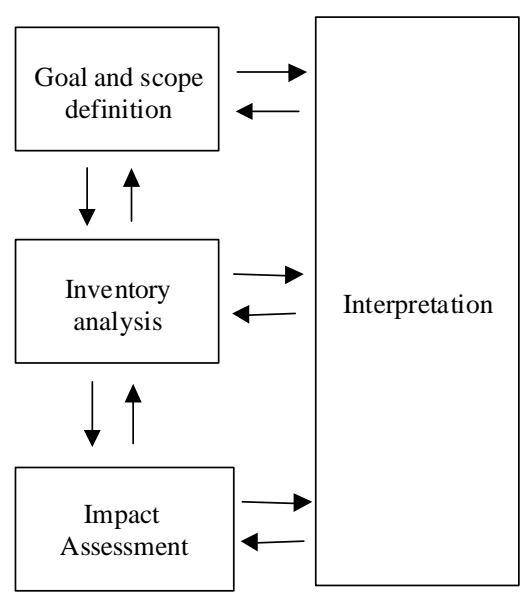

Fig. 1. The main phases of an LCA according to the ISO standard [11].

\section{Inventory Analysis}

In the inventory analysis the material and energy flows are quantified. The system consists of several processes or activities, e.g., crude material extraction, transport, production, waste handling, and in the Fire-LCA model, fires. The different processes in the system are then quantified in terms of energy use, resource use, emissions etc. Each sub-process has its own performance unit and several in- and outflows. The processes are then linked together to form the system to analyse. The final result of the model is the sum of all in- and outflows calculated per functional unit for the entire system.

\section{Impact Assessment}

The most difficult part and also the most controversial part of an LCA is the impact assessment. So far, no standard procedure exists for the implementation of an entire impact assessment. However, the ISO standard covers the so-called Life Cycle Impact Assessment (LCIA) [13], where different impact categories are used and recommendations are given for Life Cycle Interpretation [14]. Transparency of the LCA model is, however, important and inventory data must also be available in addition to aggregated data. Several methods/tools have been developed for impact assessment and the tools can usually be integrated with different LCA computer software. The modern 
tools today usually include a classification and characterisation step where the different parameters e.g., emissions are aggregated to different environmental classes such as acidification, climate change or eutrophication. There are also possibilities for direct evaluation/interpretation of the different emissions or environmental classes.

\section{THE RISK ASSESSMENT APPROACH}

In a conventional LCA the risk factors for accidental spills are excluded. In the LCA data for the production of a chemical, for example, only factors during normal operation are considered. However, there can also be, for example, emissions during a catastrophic event such as an accident in the factory. Those emissions are very difficult to estimate due to a lack of statistical data and lack of emission data during accidents. The same type of discussion exists for electric power production in nuclear power plants.

In the case of the evaluation of normal household fires the fire process can be treated as a commonly occurring activity in the society. The frequency of fire occurrences is relatively high (i.e., high enough for statistical treatment) and statistics can be found in most countries. This implies that it is possible to calculate the different environmental effects of a fire if emission factors are available. Statistical fire models can be set up for other types of fires but the uncertainty in the statistical fire model will increase as the statistical data is more limited.

The fundamental function of a better fire performance is to prevent a fire from occurring or to slow down the fire development. Improving the fire performance of a product will, therefore, change the occurrence of fires and the fire behaviour. By evaluating the fire statistics available with and without different types of fire performance enhancers, the environmental effects can be calculated. The benefits of a higher fire performance must be weighed against the "price" society has to pay for the production and handling of possible additives and/or other modes of production. The LCA methodology will be used to evaluate the application of higher fire performance in society. In this way a system perspective is applied.

\section{FIRE-LCA METHODOLOGY}

Schematically the LCA model proposed for a Fire-LCA can be illustrated as in Fig. 2. The model is essentially equivalent to a traditional LCA approach with the inclusion of emissions from fires being the most significant modification. In this model a functional unit is characterised from the cradle to the grave with an effort made to incorporate the emissions associated with all phases in the units life-cycle. Thus, the model includes production of material for the product to be analysed, as well as the production of the additives if applicable. If possible the model should be designed in such a way that the fire performance can be varied. Furthermore, the model should include production, use and waste handling of the product during its lifetime.

During the lifetime of the products to be analysed, some products will be involved in different types of fires. The Fire-LCA model will therefore include modules to describe the fire behaviour for the different types of fires. Fire statistics are used to quantify the amount of material involved in the different types of fires. In addition, the model should also include modules for handling the production of replacement materials that is needed due to the shortening of lifetime that the fires have caused. If possible the model should also include modules for the handling of the fire extinguishing process and the decontamination process. 


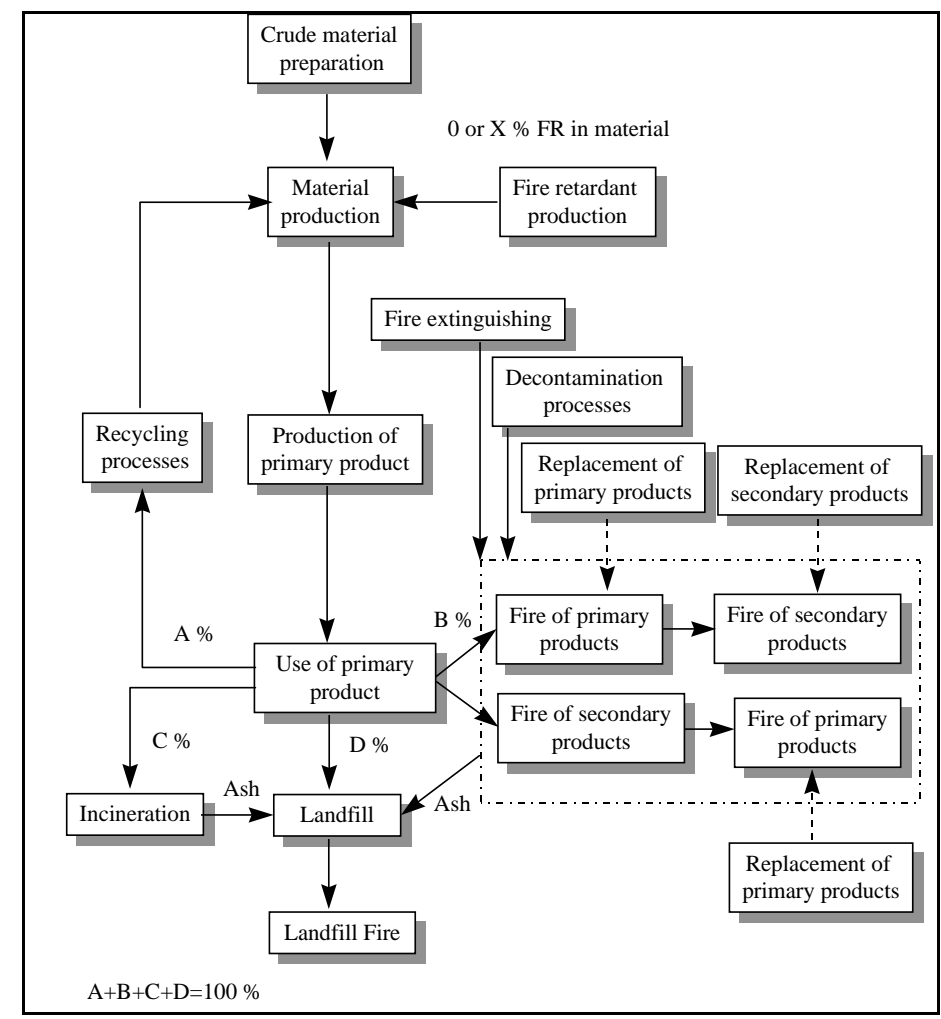

Fig. 2. Schematic representation of the LCA model.

\section{System Boundaries}

The main parts to be included in the model are:

- Production of materials and fuels to be used in the product production.

- Production of the fire protection system.

- Production of the product to be analysed (defined as the primary product).

- Use of the primary product.

- Waste handling of the primary product including:

- Landfill

- Incineration

- Recycling

- Fire modules describing:

- Fires starting at primary product and spread to surrounding products (defined as secondary products). These fires are called primary fires. Fires starting at secondary product and spread to primary product. These fires are called secondary fires.

- $\quad$ wastes from fire activities including demolition, decontamination, landfill, incineration, recycling

- Replacement of primary products lost in both primary and secondary fires. Replacement of secondary products that have been lost in primary fires spreading to secondary products. 
- Fire extinguishing activities.

- Landfill fires in the landfilled materials.

This represents a comprehensive list of the processes involved in fires. In practice it is sometimes not possible to include all of the above activities.

According to standard practice no account is taken of the production of infrastructure such as construction of plants for production of chemicals etc., or impact due to personnel. Concerning the features of the model that are specifically related to fires the system boundaries should be set such that they do not appear contrived. In general, it is realistic that we assume that material that is consumed in a fire would be replaced. Where possible, one should rely on literature data to ascertain the size of such contributions. In lieu of such data an estimate of the contribution should be made based on experience of similar systems. In the case of small home fires, which are extinguished by the occupant without professional help, the mode of extinguishment is not included due to the difficulty in determining the extinguishing agent. In cases where the fire brigade is called to a fire, transport and deployment should be included as realistically as possible. In the case studies performed so far using the Fire-LCA model, however, neither extinguishment activities nor landfill fires have been included due to lack of suitable data.

\section{STATISTICAL FIRE MODEL}

In order to include the impact of fires into the Fire-LCA model it is necessary to develop a model of the projected number of fires the functional unit would be expected to be involved in. In the Fire-LCA model two different types of fires are included: primary and secondary. Primary fires are fires that begin in the functional unit, being either confined to the functional unit or spreading beyond it. Secondary fires involve the functional unit but it is ignited only as a consequence of a large fire starting elsewhere.

In the Fire-LCA model these types of fires are treated differently. Primary fires are taken to be the responsibility of the functional unit and the full burden of fire emissions and the environmental cost of replacement of items involved in the fire are included in the LCA of the functional unit. Secondary fires are not the responsibility of the functional unit and the emissions associated with the functional unit itself and the environmental cost of replacement of the functional unit are included in the LCA but not the emissions from other items included in the fire.

This is well illustrated in the TV Case Study. In this case a model was developed based on statistics from the Swedish National Electrical Safety Board, European data compiled by the Sambrook Institute, US, data from the NFPA and insurance statistics from the Insurance Federation in Sweden [15]. In all cases the Primary fires represent fires where the fire started in the functional unit (in this case the TV set) and the Secondary fires represent fires that start elsewhere in a domestic setting.

In all cases the secondary fires are related to the house fires that are not included in the primary fires. In this model "Other Emissions" are included and refer to emissions associated with the replacement of the products in the fires. In the primary fires all products included in the fire must be replaced (although the minor fires that make up the majority of primary fires from insurance statistics are assumed to be repaired in most cases with only $30 \%$ replacement which is probably a conservative figure). In the secondary fires only the emissions for replacement of the functional unit are included in the replacement. 
Significantly it is also assumed in the model that the functional unit is replaced, on average, after $50 \%$ of its life-cycle has expired. Thus all replacement emissions are weighted into the model at $50 \%$ of the emissions associated with the production of a new functional unit.

In order to efficiently apply the Fire-LCA model it is necessary to have a detailed fire model. In some cases (as for the TV Case Study and the Furniture Case Study) a significant body of data may exist on which to base the fire model. In other cases (e.g., the Cables Case Study) some postulation of fire performance may be necessary.

The detail available in fire statistics varies between countries and sources for these statistics. In many cases it is not possible to find sufficiently detailed statistics. In these cases is prudent to run the Fire-LCA model with several assumed statistical fire models and conduct a sensitivity analysis. Further, it is imperative that the assumptions are clearly defined in the model presentation to facilitate a critical evaluation of the results.

\section{DATA INVENTORY}

In the inventory analysis the material and energy flows are quantified. An important aspect in the inventory analysis is the model resolution. The model resolution can be expressed as the smallest unit that can be resolved in the analysis. The resolution requirements are determined by the type of final evaluation. In many cases the evaluation includes an evaluation of different fire protection systems e.g., use of different flame retardants. This requires a high resolution of the composition of the product materials, usually plastic materials. The resolution and quality of the model must be so high that the composition of the materials can be varied and the result can be evaluated. This can usually be considered as a high resolution for an LCA. Furthermore all relevant emissions have to be covered as well as the use of raw materials and energy resources.

\section{Emissions from Fires}

Literature data on fire emissions can be used if available. The emissions should be rather detailed and preferably include e.g., $\mathrm{CO}, \mathrm{CO}_{2}, \mathrm{HCN}, \mathrm{NH}_{3}, \mathrm{HCl}, \mathrm{NO}_{\mathrm{x}} \mathrm{HBr}, \mathrm{VOC}$, PAHs, isocyanates, chlorinated, and brominated dioxins and furans. However, if the products evaluated do not, for example, contain any phosphorous then the phosphorous containing species can be excluded. Similarly, if the product contains any specific additives then these must be included in the measurement together with possible products when this additive burns, for example if the product contains any brominated flame retardant (FR) then the specific FR in question and brominated dioxins and furans should be considered.

If literature data is not available then experimental data should be obtained. The fire experiments should provide as realistic input data as possible to the Fire-LCA model. Preferably one should conduct at least one test for each type of fire in order to obtain a good estimate of the emissions from the fires. Fire experiments and the analyses needed to measure the emissions are, however, costly and therefore the number of experiments must be optimized. The fire experiments should give required input to the primary fires (confined to the functional unit, confined to the room of origin or confined to the house of origin) and the secondary fires (in this case relating to the emissions from the functional unit only).

Fire emissions vary depending on ignition source, amount of oxygen available and possible catalytic effects from other material in the vicinity of the product evaluated. 
However, the amount of oxygen is usually not restricted in the beginning of a fire, i.e., the experiment for data on ignition in the product evaluated can usually be conducted in normal room conditions. For cases where one can suspect that the oxygen supply might be very limited experiments can be conducted with varying oxygen supply, which was the method used in the cable case study [7]. If the ignition source is small so that the product only smoulders then the emissions are likely to be different but there is insufficient detail in the fire statistics to determine how common such fires are. For the larger fire such as when the entire room burns then the emissions measured in the case studies conducted so far have been very similar, this implies that the emissions are quite similar irrespective of material content and ignition source. There are however currently no methods to measure the emissions in a closed room fire.

\section{RESULTS AND DISCUSSION}

Results from an LCA study can be presented in numerous ways. Typically LCA studies are conducted in a comparative manner with one model of the functional unit being compared to another. In the Fire-LCA studies conducted to date the model has been used in two senses, i.e., to:

- compare two version of the same product with essentially the same components and materials, but with different fire performance, or to

- compare two versions of a product with the same overall fire performance but different construction materials.

In the first case one can draw conclusions concerning the environmental impact of choosing a high level of fire performance over a low level of fire performance. In the second case one can draw conclusions concerning the relative environmental impact of the products depending on which materials are used in the product construction, when the environmental impact of fires is included in the overall LCA treatment. Naturally in both cases the model is open to the investigation of different scenarios within the same general comparison, e.g., in terms of comparing different end-of-life scenarios or different modes of production.

The life cycle model offers many different possibilities for the analysis of the results. Each model in the LCA-software contains one scenario, e.g., four scenarios in any given study represent four different models. The analyses can be divided in two different parts: analyses of the different modules in a scenario, and comparison between different scenarios. Nearly all life cycle assessments contain a large quantity of data. Usually it is neither possible nor necessary to analyse all the different parameters in a study. It is, therefore, of great importance to select and analyse central parameters. The selection of parameters for further analysis should focus on two different criteria. Firstly, the parameter must be well represented in the system with high quality data and few data gaps, secondly data of direct relevance for the fire aspect should be placed in focus when using the Fire-LCA model. Should the data of direct relevance for the fire aspects not exhibit major differences between the different scenarios one could interpret the results to mean that the Fire part of the model does not offer any additional insight over that of a traditional LCA and the uncertainties in that part of the model should be removed by reverting to a traditional LCA treatment.

The utility of the Fire-LCA model is illustrated below using the TV Case Study. Figure 3a shows the results of a comparison between emissions of PAH from the life-cycle of a 
flame retarded TV and a non-flame retarded TV using the Fire-LCA model. In this figure a modern scenario "today" and a future scenario are compared. In both cases one can see that fires are a significant source of PAH from TVs throughout their life-cycle. This aspect would not be included in a traditional LCA analysis. Differences between the modern scenario and the future scenario are mainly due to difference in the emissions associated with energy recycling of material included in TV sets at the end of their life cycle. This can be said to be true of all major organic species whose emissions are minimized from controlled combustion but who are produced to a large degree in fires.

Similar results were found for the Furniture case study as shown in Fig. 3b. There is a significant difference in the emissions of PAH from the non-FR sofa compared to the two FR sofas, largely due to the impact of fires. In this figure one can also see that the difference between the two FR sofas is small indicating that the greatest benefit of using this model is obtained when comparing products with different fire performance.

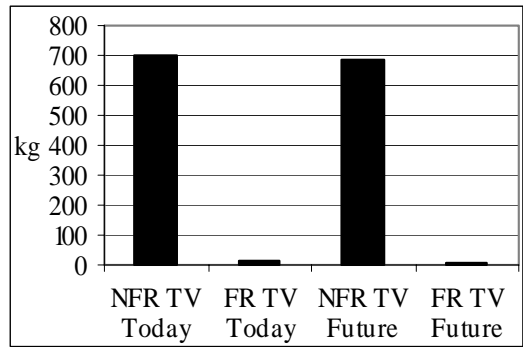

a) TV Case Study

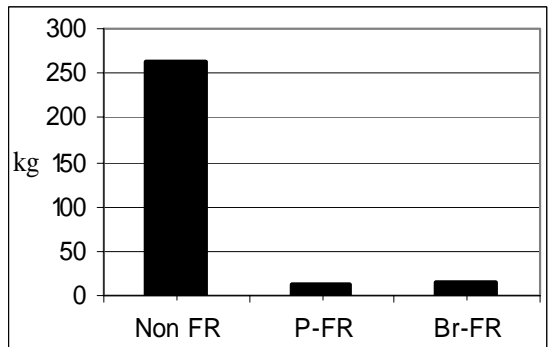

b) Furniture Case Study

Fig. 3. The main PAH emissions comes from the different fires in the system. The use of flame retardants decreases the number of fires and thus also the PAH emission.

The importance of emissions from fires on the Life-Cycle emissions of a given species are not, however, always as significant as in the example above. For species that are produced to a large degree from stationary energy production sources the difference in Life-Cycle emissions is marginal between the high fire performance and the low fire performance versions of the products studied to date. This is illustrated for carbon monoxide (CO), which is an important acute toxic gas in fires, in Fig. 4.

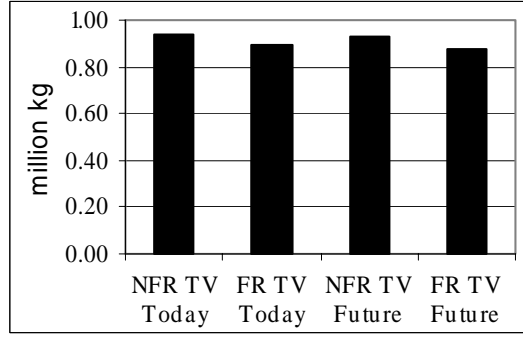

a) TV Case Study

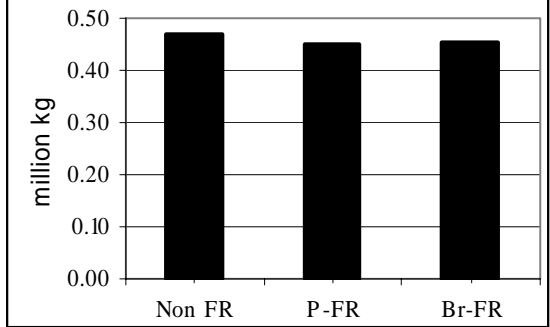

b) Furniture Case Study

Fig. 4. Carbon monoxide (CO) emissions per million functional units for the full unit life-cycle, illustrating the similarity between $\mathrm{CO}$ emissions from both the high and the low fire performance versions of the functional units. 


\section{CONCLUSIONS}

Fire-LCA is an LCA method that incorporates fires as one possible end of life scenario. It was developed in order to be able to assess life cycle aspects of the fire performance of a product.

A great deal of input data is needed in order to conduct a Fire-LCA study. Very little fire emission data is reported in the literature. Only recently have detailed characterisation of fire emissions been conducted on a more regular basis in some laboratories. Much data is confidential. However, as the number of Fire-LCA studies and research on fire emissions increase, such data will become more readily available. It can also be difficult to find production data for some materials, although this problem is common to both Fire-LCA and traditional LCA applications.

The fire statistics needed in order to set up the statistical fire model in a Fire-LCA analysis must be detailed. If the statistics is not detailed enough one has to make some assumptions and conduct a sensitivity analysis on the statistical fire model.

Ideally, when evaluating the environmental impact of the choices made to adopt a high level of fire safety one should include all the emissions available. Currently no agreed evaluation method to take into account and compare all the different emissions using the Fire-LCA Model exists. However the following general observations can be made:

- Controlling the ignitibility and growth of fires in products has a major effect on reducing toxic environmental pollutants such as $\mathrm{PAH}$.

- The significance of the difference in emissions from the whole life-cycle is greatest for large organic species (e.g., PAH). The production of these species is minimized from controlled combustion but they are produced to a large degree in fires. In contrast species (e.g., CO), which are produced to a large degree from stationary sources, exhibit only minor differences in the emissions from the whole product life-cycle.

- Which flame retardant is used to obtain a given level of fire performance has a significant potential impact on the emissions from the overall Fire-LCA treatment of that product.

- In cases where there is a significant difference in the fire performance of different versions of any given product the Fire-LCA approach must be used to obtain a realistic assessment of the environmental impact of the method used to obtain the high level of fire safety.

Finally, further modification of the Fire-LCA model is needed. This model cannot adequately deal with specific toxicity aspects of the chemicals used to improve the fire performance of a product nor can it adequately take into account the toxicity of fires. Similarly, the social or economic impact of fires, both in terms of lost work or debilitation of worker is in no way included in the model. It is hoped that future versions of the model will allow for at least some of these important aspects.

\section{REFERENCES}

[1] Simonson, M., Boldizar, A., Tullin, C., Stripple, H., and Sundqvist, J.O., "The Incorporation of Fire Considerations in the Life-Cycle Assessment of Polymeric Composite Materials: A Preparatory Study,” SP Report 1998:25, 1998. 
[2] Simonson, M., and Stripple, H., "The Incorporation of Fire Considerations in the Life-Cycle Assessment of Polymeric Composite Materials: A preparatory study,” Interflam, pp 885-895, 1999.

[3] Simonson, M., Blomqvist, P., Boldizar, A., Möller, K., Rosell, L., Tullin, C., Stripple, H., and Sundqvist, J.O., "Fire-LCA Model: TV Case Study,” SP Report 2000:13, 2000.

[4] Simonson, M., and Stripple, H., "LCA Study of TV Sets with V0 and HB Enclosure Material," Proceedings of the IEEE International Symposium on Electronics and the Environment, 2000.

[5] Simonson, M., and Stripple, H., "LCA Study of Flame Retardants in TV Enclosures,” Flame Retardants 2000, pp.159-170, 2000.

[6] Simonson, M., Tullin, C., and Stripple, H., "Fire-LCA Study of TV Sets with V0 and HB Enclosure Material,” Chemosphere, 46, pp.737-744, 2002.

[7] Simonson, M., Andersson, P., Rosell, L., Emanuelsson, V., and Stripple, H., “Fire-LCA Model: Cables Case Study,” SP Report 2001:2 available at http://www.sp.se/fire/br_reports.HTM.

[8] Simonson, M., Andersson, P., Emanuelsson, V., and Stripple, H., "A Life-cycle Assessment (LCA) Model for Cables Based on the Fire-LCA model," Fire and Materials, 27, pp.71-89, 2003.

[9] Andersson, P., Simonson, M., Rosell, L., Blomqvist, P., and Stripple, H., "FireLCA Model: Furniture Case Study,” SP report 2003:22, 2003.

[10] Andersson, P., Simonson, M., Blomqvist, P., and Stripple, H., "Fire-LCA Model: Furniture Case Study,” Flame Retardants 2004, pp 15-26, 2004.

[11] "Environmental management - Life cycle assessment - Principles and framework,” ISO 14040:1997.

[12] "Environmental management - Life cycle assessment - Goal and scope definition and inventory analysis,” ISO 14041:1998.

[13] "Environmental management - Life cycle assessment - Life cycle impact assessment,” ISO 14042:2000.

[14] "Environmental management - Life cycle assessment - Life cycle impact interpretation,” ISO 14043:2000.

[15] De Portere, M., Schonbach, C., Simonson, M., "The Fire Safety of TV Set Enclosure Materials, A survey of European statistics”, Fire and Materials, 24, 53-60, 2000. 